\title{
Technè
}

La science au service de l'histoire de l'art et de la préservation des biens culturels

$44 \mid 2016$

Archives de l'humanité : les restes humains patrimonialisés

\section{Archéoentomologie et archéoparasitologie d'une momie égyptienne}

Archaeoentomology and archaeoparasitology of an Egyptian mummy

Jean-Bernard Huchet

\section{OpenEdition}

1 Journals

Édition électronique

URL : http://journals.openedition.org/techne/1120

DOI : 10.4000/techne. 1120

ISSN : 2534-5168

Éditeur

C2RMF

Édition imprimée

Date de publication : 1 novembre 2016

Pagination : 79-83

ISBN : 978-2-7118-6339-6

ISSN : 1254-7867

Référence électronique

Jean-Bernard Huchet, «Archéoentomologie et archéoparasitologie d'une momie égyptienne », Technè [En ligne], 44 | 2016, mis en ligne le 19 décembre 2019, consulté le 23 juillet 2020. URL : http:// journals.openedition.org/techne/1120; DOI : https://doi.org/10.4000/techne.1120

\section{(c) $\Theta \Theta \Theta$}

La revue Technè. La science au service de l'histoire de l'art et de la préservation des biens culturels est mise à disposition selon les termes de la Licence Creative Commons Attribution - Pas d'Utilisation

Commerciale - Pas de Modification 4.0 International. 


\section{Jean-Bernard Huchet \\ Archéoentomologie et archéoparasitologie d'une momie égyptienne}

Archaeoentomology and archaeoparasitology of an Egyptian mummy

Résumé. En dépit des soins méticuleux apportés par les antiques thanatopracteurs égyptiens, de nombreuses momies ont subi les assauts d'insectes déprédateurs. La momie égyptienne du musée Anne-de-Beaujeu, confiée au département Restauration $d u C 2 R M F$ de Versailles pour restauration, n'a pas échappé aux vagues d'arthropodes thanatophages qui se sont préservés jusqu'à nous. Au-delà de cette colonisation post-mortem, les investigations paléoparasitologiques conduites sur cette momie nous révèlent que cette dernière souffrait d'une pédiculose sévère, comme en témoignent les myriades de poux et de lentes découvertes sur les restes de cheveux.

Mots-clés. Archéoentomologie, archéoparasitologie, insectes, Coléoptères, Diptères, ectoparasites, momie, Égypte.
Abstract. Despite the meticulous care bestowed by Ancient Egyptian embalmers, numerous mummies have suffered from attacks by depredatory insects. The Egyptian mummy from the Museum Anne-de-Beaujeu, entrusted for restoration to the C2RMF (Versailles), did not escape the invasions of thanatophagous arthropoda, which have been preserved up till now. Beyond this post-mortem colonization, paleoparasitological investigations conducted on this mummy revealed that the latter suffered from severe pediculosis, as evidenced by the myriads of lice and nits discovered on the remaining hair.

Keywords. Archaeoentomology, archaeoparasitology, insects, Coleoptera, Diptera, ectoparasites, mummy, Egypt.

\section{Introduction}

Les restes humains organiques conservés dans les musées sont les hôtes privilégiés de diverses espèces d'insectes et de parasites. L'infestation par ces animalcules peut avoir des origines distinctes : colonisation de l'individu de son vivant (ectoparasites notamment), lors des différentes étapes de la dégradation post-mortem (insectes nécrophages), ou enfin plus tardivement, au sein des collections muséales. S'agissant de momies égyptiennes, nous serions en droit d'imaginer que les divers processus de momification destinés à préserver le corps aient eu raison des divers organismes thanatophages. Il n'en est malheureusement rien. Comme en témoignent différents chapitres du Livre des Morts, les insectes étaient redoutés des embaumeurs qui mirent en œuvre toute leur ingéniosité pour éviter que ceux-ci ne viennent corrompre irrémédiablement l'intégrité des défunts dont ils avaient la charge (fig. $1 \mathrm{a}, \mathrm{b})$. D'autres preuves afférentes à cette appréhension à l'égard des thanatophages ont pu être révélées lors de séances de débandelettage, communément pratiquées au cours des deux siècles précédents. Ainsi, l'entomologiste B. Greenberg (1991) rapporte la découverte, dans la bouche d'une momie, d'un fragment de papyrus portant l'inscription suivante : «Les vers, en toi, ne se transformeront en mouches. »
Originellement destinés à préserver les corps pour l'éternité, les savoir-faire des embaumeurs ont eu comme conséquence indirecte de préserver les insectes contemporains de l'embaumement. À l'instar de leur hôte, ces fragiles reliques ont traversé les siècles. Elles nous livrent de précieuses informations sur l'état sanitaire de ces populations d'époque pharaonique et participent à la reconstitution des pratiques funéraires de l'Égypte ancienne ${ }^{1}$.

Nous présentons ici les résultats de l'étude archéoentomologique ${ }^{2}$ conduite sur une momie égyptienne (XXII ${ }^{\mathrm{e}}$ dynastie/800-750 av. J.-C.) du musée Anne-de-Beaujeu (Allier) confiée au département Restauration du C2RMF ${ }^{3}$ de Versailles pour y être traitée. De très nombreux restes d'insectes, le plus souvent remarquablement préservés, ont pu être mis au jour (fig. 5). Ces derniers sont décrits et illustrés ci-après.

\section{Déceler la présence d'insectes et de leurs traces d'activité sur des momies}

Si certains restes d'insectes se révèlent facilement décelables par les restaurateurs lors de la phase de restauration, la taille et/ou la fragmentation de certains spécimens nécessite le prélèvement de restes pulvérulents et autres échantillons de

Jean-Bernard Huchet, CNRS, Muséum national d'Histoire naturelle (UMRs 7209 et 7205) et Université de Bordeaux (UMR 5199 PACEA) (huchet@mnhn.fr). 

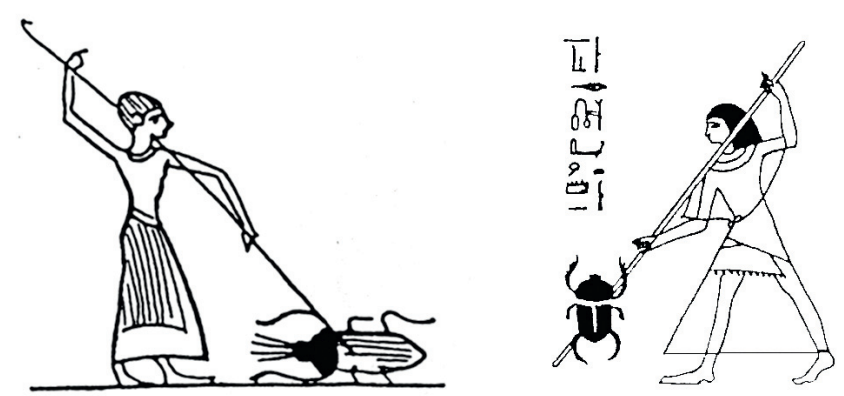

Fig. 1. Prêtre égyptien repoussant l'insecte nécrophage. Vignettes extraites du chapitre 36 du Livre des Morts (a : Papyrus Louvre N 3248 ; b : Papyrus Ryerson, modifiés).

nature organique (cheveux notamment) pour examen au laboratoire. Après avoir fait l'objet d'une série de tamisages $(5 \mathrm{~mm} / 1 \mathrm{~mm} / 300 \mu \mathrm{m})$ pour isoler les restes organiques des détritus exogènes, les fragments d'exosquelettes sont triés sous stéréo-microscope puis identifiés. Dans certains cas, le recours au microscope électronique à balayage (MEB) permet d'affiner et/ou de confirmer l'identité taxinomique des divers arthropodes en mettant en évidence certains détails morphologiques diagnostiques.

\section{Les insectes de la momie du musée Anne-de-Beaujeu}

L'examen microscopique des prélèvements effectués au C2RMF témoigne, a posteriori, d'une activité intense au sein de la momie comme l'indique le nombre considérable de déjections d'insectes et de fragments d'exuvies ${ }^{4}$. De nombreux cristaux s'apparentant à du natron, carbonate naturel de sodium utilisé par les embaumeurs pour déshydrater les corps, figuraient au sein des échantillons.

Les restes attribuables à quatre espèces, appartenant à trois ordres distincts d'insectes, ont pu être identifiés. Dans le cas présent, la biologie de ces différentes espèces et leur localisation viennent corroborer le fait que ces derniers sont contemporains de la phase de momification et non la conséquence d'une infestation au musée. Comme il nous sera donné de le voir ici, l'approche archéoentomologique fournit de précieux enseignements tant sur les processus de momification en Égypte antique que sur l'état sanitaire des populations du passé.

Parmi les taxons représentés figurent deux espèces de coléoptères, ainsi qu'une espèce de diptère (mouche nécrophage), toutes trois hôtes classiques des momies égyptiennes. Cependant, la découverte la plus remarquable est sans nul doute la présence de très nombreux restes de poux (Pediculus humanus capitis), sous leur forme adulte, ainsi que des myriades de lentes solidement attachées aux cheveux de la momie.

\section{Les coléoptères}

Necrobia rufipes (De Geer), la « Nécrobie à pattes rouges", très anciennement nommée Necrobia mummarium (la « Nécrobie des momies »)

Ce petit coléoptère (3,5-7 mm de long), d'un bleu métallique, appartient à la famille des Cleridae. Il fréquente les cadavres à un stade avancé de la dégradation post-mortem. Comme nous avons pu l'indiquer précédemment ${ }^{5}$, sa présence soutient le fait que ces différentes phases de momification duraient plusieurs semaines, en concordance avec les écrits des auteurs antiques, notamment ceux d'Hérodote ${ }^{6}$, qui mentionnait que les processus de momification se prolongeaient sur sept décades ( 70 jours). Hôte «classique » des momies, cet insecte fut originellement mentionné en 1812 par le célèbre égyptologue J.-F. Champollion : « Ce fut en examinant les mains attentivement que nous aperçûmes, dans l'interstice des doigts, plusieurs coléoptères morts, de couleur rose-violet dans tout son éclat... » Des restes de cette espèce, parfaitement préservés, furent récemment découverts dans la cavité viscérale de la momie du prêtre Namenkhet Amon (XXVe-XXVI ${ }^{\mathrm{e}}$ dynastie, $-430 /-450$ av. J.-C.) ${ }^{7}$. Aujourd'hui cosmopolite, Necrobia rufipes est parfois utilisée en entomologie médico-légale dans l'établissement de l'intervalle post-mortem (IPM) ${ }^{8}$.

\section{Dermestes frischii Kugelann (fig. $2 a, b, c, d, e$ )}

Les insectes appartenant au genre Dermestes Linné (famille des Dermestidae), étymologiquement « mangeur de peau », comptent de nombreux représentants de par le monde. Dans la nature, les adultes et leurs larves se rencontrent sous les charognes à différents stades de la décomposition post-mortem avec cependant une prédilection pour les cadavres en état de dessiccation avancé ${ }^{9}$.

En contexte archéologique, un petit nombre d'espèces figure parmi les hôtes habituels des momies égyptiennes dont notamment Dermestes frischii, découvert tant sur les linges et bandages externes qu'au sein de la momie du musée. Pour l'anecdote, cette espèce fut également retrouvée lors de l'autopsie conduite sur la momie de Ramsès $\mathrm{II}^{10}$. Eu égard à leur biologie, Dermestes et Necrobia se rencontrent fréquemment simultanément sur un même corps, lorsque ce dernier libère des acides gras volatils et autres produits de dégradation caséique $^{11}$. La compétition interspécifique exerce un effet régulateur notable sur l'accroissement des populations ${ }^{12}$. Selon Atkinson (1825), les Dermestes et Necrobia n'ont pas la possibilité de s'échapper une fois la momie bandelettée (ils parviendraient à percer quelques couches de lin, pour finalement mourir in situ).

Une exuvie d'Attagenus sp. (Dermestidae) figurait dans la matière pulvérulente située sous la tête de la momie. Son remarquable état de conservation suggère cependant qu'il s'agit d'une contamination récente. À l'instar des Dermestes cités précédemment, les espèces de ce genre figurent parmi 


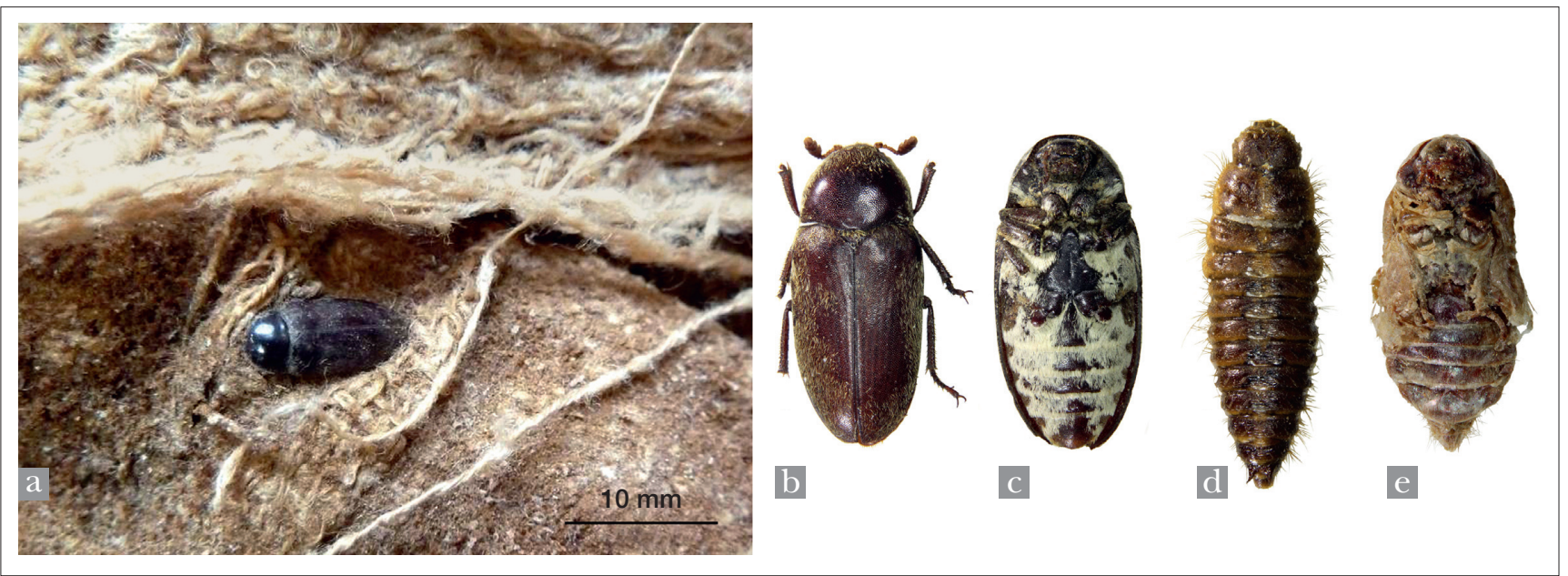

Fig. 2. Spécimens archéologiques du coléoptère Dermestes frischii (Dermestidae). a : individu adulte

découvert dans les bourres de tissus de la momie. ( C C2RMF/N. Timbart. b : adulte, vue dorsale ;

c : adulte, vue ventrale; $d$ : larve de dernier stade ; e : nymphe. $\odot \mathrm{J} .-\mathrm{B}$. Huchet/MNHN (échelles : $10 \mathrm{~mm}$ ).

les principaux ravageurs des musées dans lesquels ils occasionnent des dégâts majeurs parmi les collections d'histoire naturelle.

\section{Les mouches nécrophages}

Les diptères, sous leur forme adulte, sont des insectes relativement fragiles et se conservent plutôt mal en contexte archéologique. Toutefois, lors de la nymphose (passage de la larve à l'adulte), l'enveloppe externe de l'« asticot» se durcit et constitue un petit tonnelet rigide (puparium) à l'intérieur duquel l'insecte se métamorphose. Ces petits « cocons ", très résistants (fig. $3 \mathrm{a}, \mathrm{b}$ ), ont propension à se conserver remarquablement sur de très longues périodes. Ce sont ces derniers que l'on retrouve associés aux momies.
Les investigations conduites sur la momie du musée Annede-Beaujeu ont révélé la présence de quelques puparia d'une seule espèce de mouche aux reflets métalliques : Chrysomya albiceps.

\section{Chrysomya albiceps (Wiedemann) (fig. $3 a, b, c)$}

Chrysomya albiceps est incontestablement l'espèce nécrophage la plus fréquente en Égypte ${ }^{13}$. Sa présence aux temps pharaoniques est attestée par des découvertes récurrentes dans diverses momies d'Égypte. C. albiceps affectionne les cadavres de taille importante pour assurer la pérennité du développement larvaire. Selon G. F. Bornemissza (1957), cette espèce intervient sur les cadavres lors de la phase dite de «fermentation butyrique ${ }^{14}$ » (soit entre 20 et 40 jours après la mort). La ponte (ou oviposition) s'effectue préférentiellement au

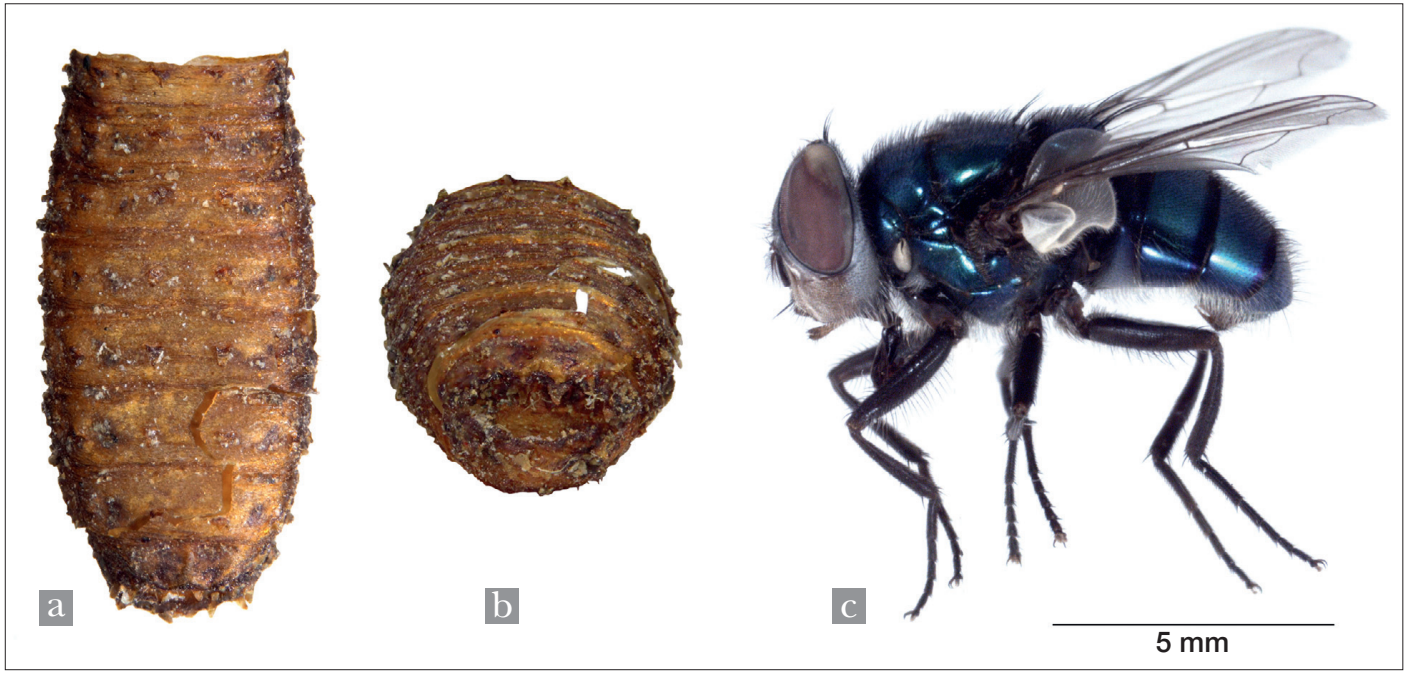

Fig. 3. La mouche nécrophage Chrysomya albiceps (Calliphoridae). a : puparium (région occipitale de la momie), vue dorsale ; $\mathrm{b}:$ idem, vue apicale, (échelle : $5 \mathrm{~mm}$ ). (c) J-B. Huchet/MNHN ; c: spécimen adulte actuel (échelle : $5 \mathrm{~mm}$ ). (C) K. Szpila, Nicolaus Copernicus University, Toruń, Pologne. 


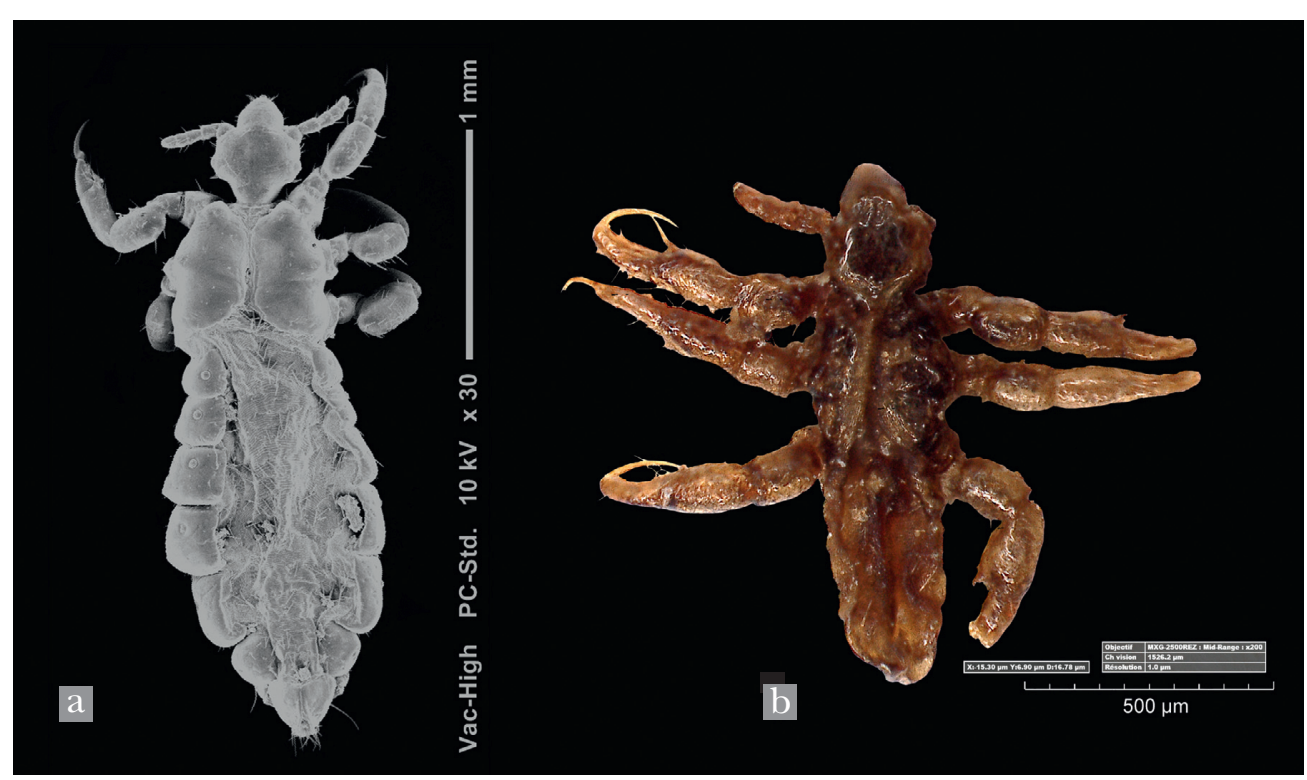

Fig. 4. Poux de tête (Pediculus humanus capitis ) provenant de la momie. a : adulte, photo MEB (microscope électronique à balayage) ;

b : nymphe. (C) C2RMF/

D. Robcis ; M. Lemoine,

Laboratoire d'Archéozoologie/ Archéobotanique du MNHN.

niveau de plaies ouvertes lorsqu'elles existent. Il est probable que les éviscérations conduites par les embaumeurs sur les corps destinés à être momifiés aient été particulièrement attractives pour les jeunes larves qui, par ce biais, ont pu alors facilement coloniser les viscères. Dans le cas présent, le nombre restreint de puparia mis au jour suggère que le corps a été soigneusement lavé après la phase de dessiccation au natron. Les expérimentations conduites en Égypte par Tantawi et coll. (1996) ont montré que l'espèce était principalement active en saison chaude, ce qui suggérerait que la mort du sujet conservé au musée Anne-de-Beaujeu ait pu intervenir durant cette période de l'année.

\section{Les ectoparasites (poux)}

Pediculus humanus capitis (De Geer), le "pou de tête "

Si l'on excepte les puces, parasites temporaires à forte mobilité, trois ectoparasites spécifiques peuvent infester l'être humain : le pou du corps (Pediculus humanus humanus L.), le pou de la tête (Pediculus humanus capitis De Geer) (fig. 4 a, b) et le pou du pubis (Phtirus pubis L.) $)^{15}$.

Contrairement aux poux du corps, les poux de tête ne sont vecteurs d'aucune maladie. Ils occasionnent cependant une nuisance physique réelle : le «prurit ». Dans la plupart des cas, le nombre d'adultes n'excède pas la dizaine sur une même tête. Toutefois, dans quelques rares cas, on a pu en dénombrer jusqu'à un millier. Les poux de tête figurent au sein de la vermine mentionnée dans la Bible comme la troisième plaie d'Égypte et on trouve trace, dans le papyrus Ebers $^{16}$, d'une recette à base de farine de dattes et d'eau destinée au traitement de la pédiculose ${ }^{17}$. Hérodote (L'Enquête, Livre II) indique que les prêtres égyptiens se préservaient des infestations de la vermine en se rasant entièrement le crâne, mais également les cils et sourcils. Cet usage semble s'être ensuite plus ou moins répandu, notamment au sein des familles de haut rang. L'archéologie nous montre cependant que cette pratique récurrente du rasage n'a pu totalement endiguer la propagation du parasite.

Fig 5. Tableau récapitulatif des insectes de la momie égyptienne du musée Anne-de-Beaujeu.

\begin{tabular}{|l|l|l|l|l|l|}
\hline Ordre & Famille & Espèce & Stades* & Nombre de restes & NMI* \\
\hline Coleoptera & Dermestidae & Dermestes frischii & A, N, L & 85 & $\mathbf{3 3}$ \\
\hline Coleoptera & Dermestidae & Attagenus sp. & L & 1 & $\mathbf{1}$ \\
\hline Coleoptera & Cleridae & Necrobia rufipes & A & 3 & $\mathbf{3}$ \\
\hline Diptera & Calliphoridae & Chrysomya albiceps & P & 4 & $\mathbf{4}$ \\
\hline Pthiraptera & Pediculidae & Pediculus humanus capitis & A, N, Le & A, N : 38; Le $:>300)$ & A, N : 37; Le : > 300 \\
\hline
\end{tabular}

* Légende : A : adulte ; N : nymphe ; L : larve ; P : puparia ; Le : lentes ;

NMI : Nombre Minimal d'Individus.

Ne sont cependant pas incluses dans ce décompte les centaines de lentes présentes sur les cheveux. 
Bien que la découverte de poux soit relativement peu fréquente en contexte archéologique, la momie du musée Anne-de-Beaujeu n'est pas la première à attester de la présence de ce parasite en Égypte antique. La première étude paléoparasitologique faisant état de la présence de poux sur des momies égyptiennes est due à Ruffer (1921). De même, de nombreux peignes à poux d'époque pharaonique ont été mis au jour (notamment à Antinoé), certains présentant encore, entre leurs dents, des vestiges de parasites ${ }^{18}$.

Par l'ampleur de l'infestation caractérisant la momie du musée Anne-de-Beaujeu (des centaines de lentes et 37 nymphes et adultes découverts sur les cheveux), le "sujet momifié » présentait incontestablement une parasitose sévère qui, par voie de conséquence, devait se traduire par un prurit majeur, sans doute généralisé à l'ensemble du scalp. L'examen attentif du crâne (notamment de la région occipitale) n'a cependant livré aucune trace d'aire lésionnelle, expression d'un grattage chronique lié une infestation parasitaire prolongée ${ }^{19}$.

\section{Conclusion}

Les procédés, aussi multiples qu'originaux, mis en œuvre par les antiques thanatopracteurs, ont rarement été en mesure d'annihiler les méfaits insidieux de la vermine sur les restes organiques humains. L'étude archéoentomologique conduite sur la momie égyptienne du musée Anne-de-Beaujeu nous révèle l'identité de ces insectes et parasites sur lesquels baumes et autres onguents n'ont eu que peu d'effet sur l'activité des cohortes d'arthropodes nécrophages. Au-delà de l'aspect forensique mettant en évidence la durée des traitements funéraires pour cette période, la présence d'insectes ectoparasites nous renseigne sur l'état sanitaire des populations de l'Égypte ancienne.
Notes

1. Huchet, 1995, $2010 \mathrm{a}, \mathrm{b}$.

2. L'archéoentomologie, branche de l'archéozoologie, est la discipline étudiant les insectes provenant de contextes archéologiques.

3. Centre de Recherche et de Restauration des Musées de France.

4. Enveloppe chitineuse abandonnée par les arthropodes lors de la mue.

5. Huchet, 2010

6. Hérodote : L'Enquête, 85-89.

7. Huchet, 2010.

8. Arnaldos et al., 2004 ; Gennard, 2007.

9. Byrd et Castner, 2009.

10. Steffan, 1982.

11. Turchetto et al., 2001.

12. Odeyemi, 1997.

13. Omar, 1995.

14. Formation d'acide butyrique par dégradation du sucre, de l'acide lactique ou de l'amidon lors de la décomposition.

15. Le papyrus Ebers, daté d'environ 1550 avant J.-C., est l'un des plus anciens traités médicaux.

16. Huchet, 2015.

17. Leca 1971.

18. Palma 1991 ; Mumcuoglu, 2008.

19. Capasso et al., 1997.

\section{Bibliographie}

Arnaldos M. I., Sánchez F., Álvarez P., García M. D., 2004, "A forensic entomology case from the Southeastern Iberian Peninsula”, Aggraval 's Internet Journal of Forensic Medicine and Toxicology 5(1), p. $22-25$.

Atkinson J., 1825, (sans titre), Transactions of the linnean Society of London 14 (3), p. 585-586.
Byrd J. H., Castner J. L., 2009, Forensic entomology: the utility of arthropods in legal investigations, 2nd ed. CRC press, Boca Raton ( $\mathrm{Fl})$.

Capasso L., Caramiello S., La Verghetta M., Di Domenicantonio L., Anastasio R.D. 1997, "Lice and related skull lesions in a female subject from Herculaneum (79 AD, South Italy)", Journal of Paleopathology 9 (3), p. 125-135.

Champollion-Figeac J.-J., 1812, « Note sur une nouvelle espèce d'Insectes du genre Corynetes de Fabricius, observée à Grenoble », Grenoble, décembre 1812, Archives départementales de l'Isère, Fonds Champollion, vol. 3, document $n^{\circ} 296$.

Greenberg B., 1991, "Flies as Forensic Indicators", Journal of Medical Entomology 28 (5), p. 565-577.

Gennard D.E., 2007, Forensic entomology: an introduction, John Wiley \& Sons Ltd, Chichester, West Sussex.

Hérodote, L'enquête, Barguet Andrée (éd. \& trad.) (1964), Paris, Gallimard.

Huchet J.-B. 1995, « Insectes et momies égyptiennes ", Bulletin de la Société linnéenne de Bordeaux 23(1), p. 29-39.

Huchet J.-B. 2010a, «Des insectes, des momies : l'apport de l'entomologie à l'étude des pratiques funéraires des sociétés anciennes ", in I. Cartron, D. Castex, P. Georges, M. Vivas \& M. Charageat (dir.), De Corps en Corps, traitement et devenir du cadavre. Publication de la MSHA, «Identité(s) et Mémoire(s) des Populations du Passé », p. 33-55.

Huchet J.-B., 2010b, "Archaeoentomological study of the insects remains found within the mummy of Namenkhet Amon, San Lazzaro Armenian Monastery, Venice/Italy", Advances in Egyptology 1, p. 58-80.
Huchet J.-B., 2015, « Ectoparasites de l'Homme : le regard de l'Archéoentomologiste ", in F. Collard et E. Samama (dir.), Poux, puces et punaises, la vermine de l'homme, découverte, descriptions et traitements. Antiquité, Moyen Âge, Temps Modernes, éditions l'Harmattan, Paris, p. 45-60.

Mumcuoglu K. E., 2008, "The louse comb: past and present", American Entomologist, 54, p. 164-166.

Odeyemi O., 1997, "Interspecific competition between the beetles Dermestes maculatus De Geer and Necrobia rufipes De Geer on dried fish", Insect Science and Its Application 17, p. 213-220.

Omar A.H., 1995, "Studies on Chrysomyia albiceps (Wiedemann), one of the most important carrion flies in Egypt", Journal of the Egyptian Society of Parasitology 25 (3), p. 607-624.

Palma R., 1991, "Ancient head lice in a wooden comb from Antionë, Egypt”, The Journal of Egyptian Archaeology 77, p. 194.

Ruffer M. A., 1921, Studies on paleopathology of Egypt, University of Chicago Press, Chicago.

Steffan J.-R., 1982, « L'entomofaune de la Momie de Ramses II », Annales de la Société entomologique de France 18 (4), p. 531-537.

Tantawi Tarek I., El-Kady Essam M., Greenberg B., El-Ghaffar Heyadat A., 1996, "Arthropod succession on exposed rabbit carrion in Alexandria, Egypt", Journal of Medical Entomology 33 (4), p. 566-580.

Turchetto M., Lafisca S. and Constantini G., 2001, "Post mortem interval (PMI) determined by study of sarcophagous biocenoses: three cases from the province of Venice (Italy)", Forensic Science International 120, p. 28-31. 